\title{
Theoretical estimation of the mechanical performance of traditional mortise-tenon joint involving a gap
}

\author{
Keita Ogawa $^{1} \cdot$ Yasutoshi Sasaki $^{1} \cdot{\text { Mariko } \text { Yamasaki }^{1}}^{1}$
}

Received: 1 September 2015 / Accepted: 3 February 2016/Published online: 11 March 2016

(C) The Japan Wood Research Society 2016

\begin{abstract}
The mortise-tenon joint, which connects columns and beams of a wooden building, often creates a gap in contact part of members. This gap is considered to affect mechanical performance of the mortise-tenon joint. This study derived a method of theoretical estimation with a gap as parameter for the mechanical performance of mortisetenon joint. In addition, it experimentally validated the method of estimation and numerically analyzed the influence of a size of such gap on mechanical properties. As a result, the estimated relationship between moment resistance and deformation angle of column shows the agreement with the experimental results, demonstrating validity of the estimation method derived. Results of the numerical analysis quantify the influence of the size of the gap on mechanical properties of the joint. The numerically analysis clarified the large influence of the gap at joints on the mechanical properties.
\end{abstract}

Keywords Wood-to-wood joint - Mechanical modeling · Moment-deformation angle relationship · Gap

\section{Introduction}

Wood-to-wood joints are common among traditional wooden framework buildings in Japan and other countries. Many of them resist an external force by engagement of their members. Therefore, the main mechanism of resistance is compression perpendicular to grain and friction

Yasutoshi Sasaki

gasteig@agr.nagoya-u.ac.jp

1 Graduate School of Bioagricultural Sciences, Nagoya University, Nagoya 464-8601, Japan between members. It is known that the rotational stiffness of the joints is rather small, however, their deformability is very large. The mortise-tenon joint covered in this study is one of such wood-to-wood joints. The joint is often used to connect columns and beams in traditional Japanese wooden buildings. The joint, of which shape is as shown in Fig. 1, is formed by inserting a tenon at the tip of column into a mortise.

Such joints play an important role in the mechanical performance of traditional wooden framework buildings. This is verified by a shaking table test for buildings. Suzuki and Maeno [1], for example, conducted a full-scale shaking table test and static test and clarified the importance of Nuki joint (crisscross joint connecting columns and beams) for the mechanical performance of buildings. Furthermore, Wang et al. [2] performed a half-scale shaking table test for a Chinese traditional wooden building to show the importance of the energy absorption capacity of mortise-tenon joints for the mechanical performance of buildings.

Therefore, the mechanical performance and evaluation of the wood-to-wood joints is a major matter of concern, and a large number of studies have been carried out for it. With regard to the mechanical performance of Nuki joints mentioned above, Guan et al. [3] simulated the relationship between moment resistance and deformation angle of column of the joints through the three-dimensional finite element model (FEM) analysis, and demonstrated that the analysis results recreated the experimental results with a high degree of accuracy. Chang et al. [4] developed a mechanical model by applying the compressive and frictional theories of wood for Nuki joints with gap, of which shape are found mainly in Taiwan, and theoretically derived an estimating equation for the relationship between moment resistance and deformation angle of column. The estimating equation was validated by its comparison with 


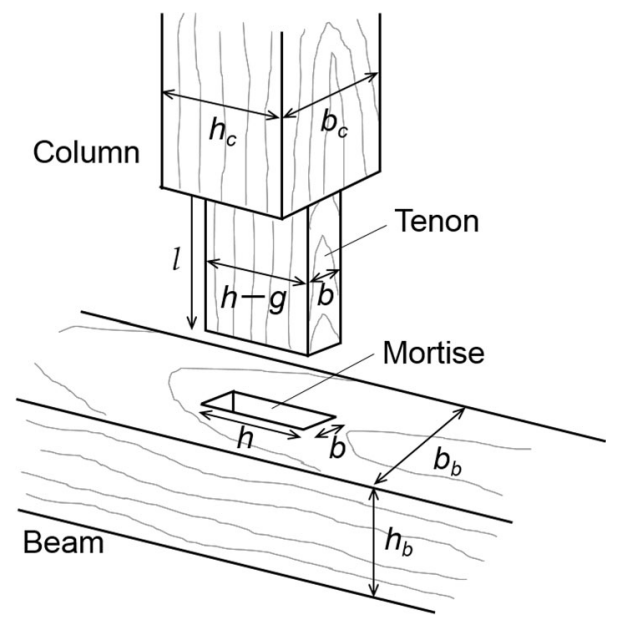

Fig. 1 Mortise-tenon joint

test results. In addition, they used the equation to simulate the relationship between gap size and rotational stiffness. The simulation shows that the involvement of gaps decreases the rotational stiffness, while its influence is limited in the range of small gap size

With regard to the mechanical performance of mortisetenon joints, Eckelman and Haviarova [5], for example, experimentally studied the influence of a size of tenon on the stiffness and maximum load, and clarified the large influence of the long-side direction length of tenon section on rotational stiffness. In addition, they demonstrated, by observing the specimens after the test, the contact between mortise and upper and lower part of tenon as well as between corner of column and beam (Fig. 2) when the column are at an angle. Nishimura et al. [6] experimentally measured the stress transfer of tenons under the rotational deformation of the joint, and examined specific features required of a mechanical analysis model. Furthermore, Sakata et al. [7] developed a mechanical model by applying the compressive and frictional theories of wood, and formulated the relationship between moment resistance and deformation angle of column at mortise-tenon joints. They validated the formulation derived through its comparison with test results.

Thus, many have studied the mechanical performance of mortise-tenon joints; however, a gap at the joints is, in fact, often caused by wood shrinkage and/or loading history. It is expected that a gap created at the joints will result in insufficient contact between members, which will affect the mechanical performance. In spite of this, a study on such gap at the mortise-tenon joints and the mechanical performance has not been conducted sufficiently. This study, therefore, examines the influence of the gaps on the mechanical performance of mortise-tenon joints. First, the relationship between moment resistance $M$ and

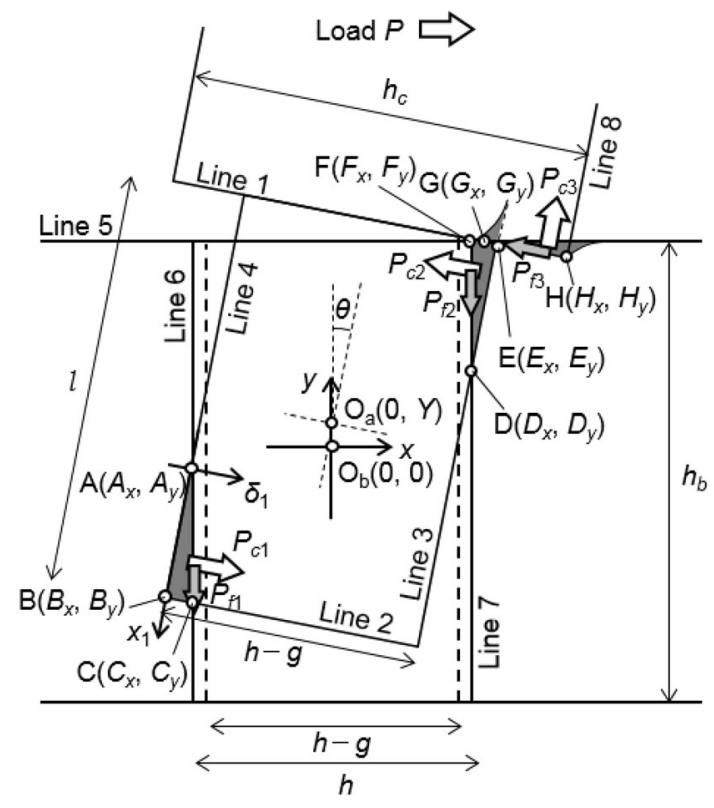

Fig. 2 Modeling of deformation at mortise-tenon joints. The joint deformed at deformation angle $\theta$ of column due to external force $P$. Partial compressive deformation are represented with the gray parts. Partial compressive and frictional resistance is represented with white and gray allows, respectively. Lines $1-8$ and Intersections A-H are defined for presenting the geometric arrangement

deformation angle $\theta$ of column at the mortise-tenon joints involving a gap is theoretically derived, and the experiments are also conducted to validate the theory. Furthermore, the method of estimation derived is used to numerically analyze the influence of a gap size on the mechanical performance. The analysis results are then compared with experimental results.

\section{Theoretical derivation}

\section{Model of joint deformation}

This study theoretically derived an equation of relationship between moment resistance $M$ and deformation angle $\theta$ of column at the mortise-tenon (column-beam) joint involving a gap. The equation enables to estimate the relationship between $M$ and $\theta$ of the joint by using the relationship between compressive stress and strain perpendicular to grain of wood. Figure 2 presents how the mortise-tenon joint rotated due to an external force $P$ applied horizontally, resulting in the deformation angle $\theta$ of column. The assumption is that the long side length $(h-g)$ of a tenon section is shorter than the one of a mortise section $h$ by $g$, leading to a gap at the mortise-tenon joint that exists on both sides with a size of $g / 2$. The mechanical model assumes that a compressive deformation locally appears 
perpendicular to the grain, which is shown in gray color in Fig. 2. In addition, $P_{c 1}, P_{c 2}$ and $P_{c 3}$ with white arrows represent the partial compressive resistance while $P_{f 1}, P_{f 2}$ and $P_{f 3}$ with gray arrows represent the frictional resistance associated with it.

As Fig. 2 shows, the deformed mortise-tenon joint is modeled in the $x-y$ plane. The model assumes that a rotational center of the joint is always at the centroid of the tenon. Therefore, the Point $O_{\mathrm{b}}$, which is regarded as an origin in the $x-y$ plane, is the center of rotation until the start of deformation. It was assumed that the column would rise with intersection $H$ as fulcrum along with increasing deformation, and it was considered that this would involve the shift of the rotational center to Point $O_{\mathrm{a}}$. The shift was presumed to occur on the $y$-axis, and the shift distance $Y$ is represented by Eq. (1):

$Y=\frac{h_{c}}{2} \sin \theta$

Although the center of rotation actually also shifts toward the $x$-axis, it is known that the amount of shift is small compared with the one along the $y$-axis [8]. Therefore, the shift was not taken into consideration here.

Second, the geometric arrangement of tenons under deformation angle $\theta$ is presented in Fig. 2. Eight lines (Lines 1-8) comprising the joint, and eight intersections (Intersections $\mathrm{A}-\mathrm{H}$ ) are shown in the figure. First of all, a linear function equation was derived that represents each Line in the $x-y$ plane with Point $O_{\mathrm{b}}$ as origin. As the slope of Line 1 is $-\tan \theta$ and $y$-intercept is $l /(2 \cos \theta)+Y$, the linear equation is as follows:

Line 1: $y=-\tan \theta \cdot x+\frac{l}{2 \cos \theta}+Y$

Similarly, the other lines (Lines 2-8) were expressed by a linear function. In addition, Intersections $\mathrm{A}-\mathrm{H}$ in Fig. 2 were calculated with the function equations for those Lines. Function equations of Lines and calculations of coordinates of each Intersections are shown in "Appendix".

\section{Compressive and frictional resistance}

Then, the compressive resistance $P_{c 1}$ was calculated that occurred at the lower part of tenon. As Fig. 2 shows, coordinate axes $x_{1}-\delta_{1}$ with Point $\mathrm{A}\left(A_{x}, A_{y}\right)$ as origin were newly established. As Axis $x_{1}$ fully overlaps Line 4, and Axis $\delta_{1}$ is perpendicular to Axis $x_{1}$, these axes rotate with a change in the deformation angle $\theta$ of column. The amount of compressive deformation at the lower tenon perpendicular to grain is represented by Eq. (3) in accordance with Axes $x_{1}-\delta_{1}$.
$\delta_{1}=\frac{\sqrt{\left(C_{x}-B_{x}\right)^{2}+\left(C_{y}-B_{y}\right)^{2}}}{\sqrt{\left(A_{x}-B_{x}\right)^{2}+\left(A_{y}-B_{y}\right)^{2}}}$

In addition, the strain $\varepsilon_{1}$ at minute intervals is obtained by dividing the deformation amount $\delta_{1}$ by the long side length $h$ of a tenon section to obtain the stress $\sigma_{1}$ at minute intervals on the $x_{1}$. The stress $\sigma_{1}$ was multiplied by the short side length $b$ of a mortise section to obtain the resistance $\Delta P_{c 1}$ at minute intervals on the $x_{1}$. This is represented by Eq. (4) by expressing the relationship between compressive stress and strain perpendicular to grain of wood as $\sigma=f(\varepsilon)$ :

$\Delta P_{c 1}=b \sigma_{1}=b f\left(\frac{\delta_{1}}{h}\right)=b f\left(\varepsilon_{1}\right)$

This is integrated by $x_{1}$ to obtain $P_{c 1}$ :

$P_{c 1}=\int_{0} \sqrt{\left(A_{x}-B_{x}\right)^{2}+\left(A_{y}-B_{y}\right)^{2}} \Delta P_{c 1} d x_{1}$

Furthermore, the frictional resistance $P_{f 1}, P_{f 2}$ and $P_{f 3}$ is represented by Eq. (6), as the normal force is multiplied by the frictional coefficient $\mu$ based on Coulomb's law:

$P_{f 1}=\mu P_{c 1} \cos \theta, \quad P_{f 2}=\mu P_{c 2} \cos \theta, \quad P_{f 3}=\mu P_{c 3}$

\section{Moment resistance at joint}

When an external force is applied on the mortise-tenon joint, the compressive and frictional resistance occurs. This study derived the relationship between $M$ and $\theta$ at the joint based on a concept that the horizontal and vertical forces are, respectively, balanced and that the moment created by an external force is balanced with the one by compressive and frictional resistance.

Here, the distance was calculated between a line of action of two types of (compressive and frictional) resistance mentioned above and the rotational center $O_{\mathrm{a}}(0, Y)$. The distance $L_{c 1}, L_{c 2}$, and $L_{c 3}$ between partial compressive resistance $P_{c 1}, P_{c 2}$, and $P_{c 3}$ and rotational center is:

$L_{c 1}=\frac{l}{2}-\frac{1}{3} \sqrt{\left(B_{x}-A_{x}\right)^{2}+\left(B_{y}-A_{y}\right)^{2}}$

$L_{c 2}=\frac{l}{2}-\frac{1}{3} \sqrt{\left(E_{x}-D_{x}\right)^{2}+\left(E_{y}-D_{y}\right)^{2}}$

$L_{c 3}=\frac{h_{c}}{2}-\frac{1}{3} \sqrt{\left(G_{x}-H_{x}\right)^{2}+\left(G_{y}-H_{y}\right)^{2}}$

The distance $L_{f 1}, L_{f 2}$, and $L_{f 3}$ between frictional resistance $P_{f 1}, P_{f 2}$, and $P_{f 3}$ and rotational center is: 
$L_{f 1}=\frac{h}{2}$

$L_{f 2}=\frac{h}{2}$

$L_{f 3}=\frac{l}{2}$

Here, the following three Eqs. (13)-(15) are derived based on the balance of horizontal and vertical forces, respectively, and that of moment with Point $O_{\mathrm{a}}$ as center, as shown in Fig. 2.

$P_{f 1}+P_{c 1} \sin \theta+P_{f 2}-P_{c 2} \sin \theta-P_{f 3} \sin \theta-P_{c 3} \cos \theta=0$

$P_{c 1} \cos \theta-P_{c 2} \cos \theta-P_{f 3} \cos \theta+P_{c 3} \sin \theta+P=0$

$$
\begin{aligned}
& P_{c 1} \cdot L_{c 1}+P_{f 1} \cos \theta \cdot L_{f 1}+P_{c 2} \cdot L_{c 2}-P_{f 2} \cos \theta \cdot L_{f 2} \\
& \quad+P_{c 3} \cdot L_{c 3}+P_{f 3} \cdot L_{f 3}=P L
\end{aligned}
$$

While these equations are similar to those suggested by Sakata et al. [7], this study differs in that it takes into consideration the deformation angle $\theta$ of column for the resistance $P_{c 1}, P_{c 2}, P_{c 3}$ and $P_{f 3}$.

Based on Eq. (6), Eqs. (13)-(15) above are:

$$
\begin{aligned}
& P_{c 1}(\mu \cos \theta+\sin \theta)+P_{c 2}(\mu \cos \theta-\sin \theta) \\
& \quad-P_{c 3}(\mu \sin \theta+\cos \theta)=0 \\
& P_{c 1} \cos \theta-P_{c 2} \cos \theta-P_{c 3}(\mu \cos \theta-\sin \theta)+P=0 \\
& P_{c 1}\left(L_{c 1}+L_{f 1} \mu \cos ^{2} \theta\right)+P_{c 2}\left(L_{c 2}-L_{f 2} \mu \cos ^{2} \theta\right) \\
& +P_{c 3}\left(L_{c 3}+L_{f 3} \mu\right)=P L
\end{aligned}
$$

Furthermore, by organizing Eqs. $\left(13^{\prime}\right)-\left(15^{\prime}\right)$, the relationship between $M$ and $\theta$ at the mortise-tenon joints is expressed by Eq. (16).

$M=P L=\frac{p(\theta) \cdot t(\theta)-q(\theta) \cdot s(\theta)}{r(\theta) \cdot t(\theta)-q(\theta) \cdot u(\theta)} P_{c 1} L$,

where

$$
\begin{aligned}
p(\theta)= & \cos ^{2} \theta\left(\mu^{2}-1\right)-\mu \sin \theta \cos \theta-\sin ^{2} \theta \\
q(\theta)= & \cos ^{2} \theta\left(\mu^{2}+1\right)-\mu \sin \theta \cos \theta+\sin ^{2} \theta \\
r(\theta)= & \mu \sin \theta+\cos \theta \\
s(\theta)= & \left(L_{c 1}+L_{f 1} \mu \cos ^{2} \theta\right)(\mu \cos \theta-\sin \theta) \\
& +\left(L_{c 3}+L_{f 3} \mu\right) \cos \theta \\
t(\theta)= & \left(L_{c 2}-L_{f 2} \mu \cos ^{2} \theta\right)(\mu \cos \theta-\sin \theta) \\
& -\left(L_{c 3}+L_{f 3} \mu\right) \cos \theta \\
u(\theta)= & L(\mu \cos \theta-\sin \theta)-\left(L_{c 3}+L_{f 3} \mu\right)
\end{aligned}
$$

\section{Materials and methods}

\section{Mechanical test with joint specimens}

In order to validate the estimation method derived in this study, a mechanical test was conducted on mortise-tenon joints. The six specimens, which are T-shaped, consisting of column and beam (Fig. 3) were prepared. Columns are made of Japanese cedar (Cryptomeria japonica D.DON) $1300 \mathrm{~mm}$ long and beams are made of Japanese cypress (Chamaecyparis obtusa Sieb. et Zucc.) $800 \mathrm{~mm}$ long, both of which are air-dried wood. The section size of columns and beams $\left(b_{c} \times h_{c}\right.$ and $\left.b_{b} \times h_{b}\right)$ is $105 \times 105 \mathrm{~mm}$, and the size of tenons is $90(l) \times 68-72(h-g) \times 30(b) \mathrm{mm}$. As Fig. 1 shows, the long side length $(h-g)$ of a tenon section was processed to be slightly smaller than that of a mortise $h$ to provide a gap $g$ at the joint $(g=2.98,2.96$, $2.83,2.48,1.02$, and $0.99 \mathrm{~mm}$ ).

The test with the joint specimens was conducted in accordance with the test method standardized by Japan Housing and Wood Technology Center [9]. Figure 3 shows test specimen and experimental setup. The beam of specimen was secured to a load test device with anchor bolts, while a tip of the column was pin-jointed to a hydraulic actuator through an iron jig and load cell. With this as loading point, the moment resistance at the joints created by the reverse cyclic load was measured. The loading sequence consisted of ten cyclic steps with three identical cycles. The deformation angle amplitudes $\theta$ of each of the cyclic steps were $\pm 1 / 450,1 / 300,1 / 200,1 / 150,1 / 100,1 / 75$, $1 / 50,1 / 30,1 / 10$ and 1/6.5 rad (at the maximum stroke of the machine). Two displacement gages were set on the upper and lower parts of column, and the deformation angle $\theta$ of column was obtained based on values indicated by the gages $v_{1}$ and $v_{2}$ and distance between the gages $d$

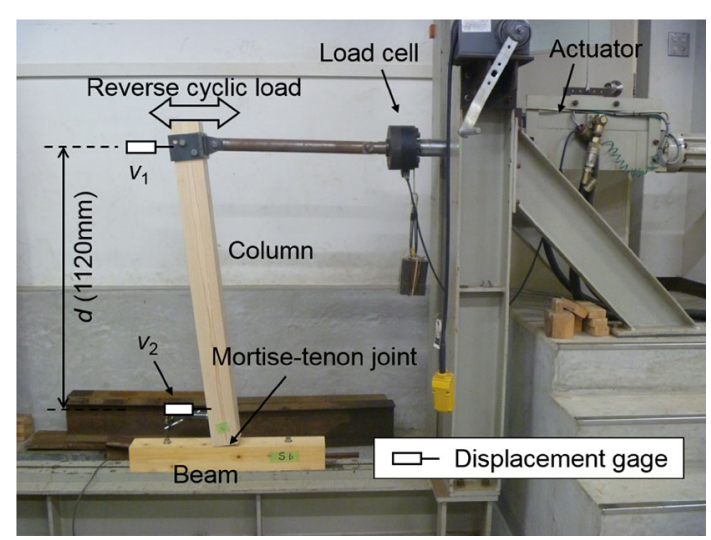

Fig. 3 Experimental setup of mechanical test with mortise-tenon joint 
$(1120 \mathrm{~mm})$. If the bending deformation of column was small enough to ignore, the deformation angle $\theta$ could be simply represented by Eq. (17):

$\theta=\frac{v_{1}-v_{2}}{d}$

As the movement speed of actuator is $100 \mathrm{~mm} / \mathrm{min}$, the test time was around 55 min per specimen.

\section{Compression perpendicular to grain test with small clear specimens}

The estimation method derived in this study requires the relationship between compressive stress and strain perpendicular to grain $[\sigma=f(\varepsilon)$ in Eq. (4)]. Therefore, small clear specimens were taken from columns of the joint specimens to conduct the test. Six small clear specimens, whose size is $30 \times 30 \times 90 \mathrm{~mm}$, were obtained from each column of the six T-shaped specimens, totaling 36. As Fig. 4 shows, loading plates were placed on upper and lower surfaces of the specimen [10], and the loading area was $30 \times 30 \mathrm{~mm}$, and the length of indirectly loaded area on both sides of the loading part was $30 \mathrm{~mm}$, respectively. A load was applied in a radial direction of wood. The loading speed of crosshead is $1.0 \mathrm{~mm} / \mathrm{min}$. The relationship between stress $\sigma$ and strain $\varepsilon$ is approximated by Eq. (18) [11] to determine a parameter that expresses this relationship. Then, Eq. (18) is assigned to Eq. (4), and finally Eq. (16) allows calculation of the relationship between moment resistance $M$ and deformation angle $\theta$ of column at joints:

$\sigma=f(\varepsilon)=\left(m_{0}+m_{1} \cdot \varepsilon\right)\left[1-\exp \left\{-\frac{E_{\perp}}{m_{0}}\left(\varepsilon+\alpha \cdot \varepsilon^{\beta}\right)\right\}\right]$

Here, $E_{\perp}$ represents an elastic coefficient under compressive load perpendicular to grain, $m_{0}$ represents an

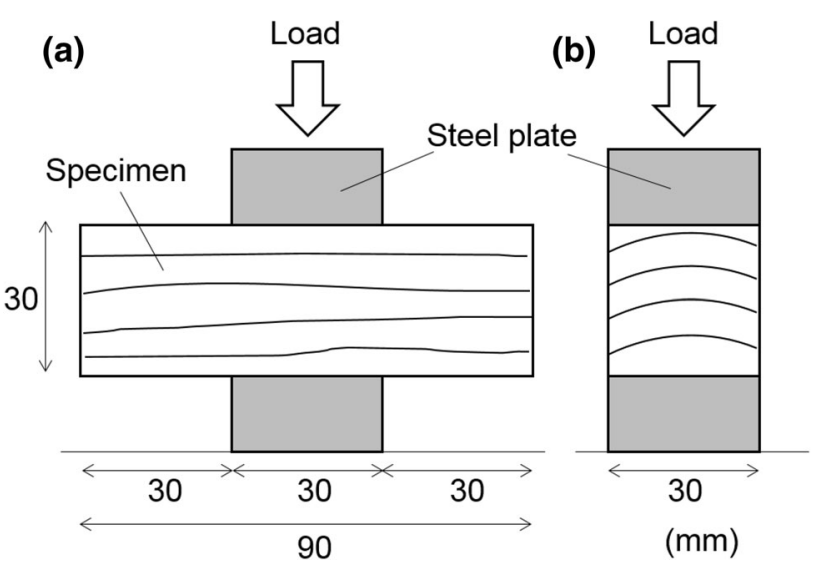

Fig. 4 Compression perpendicular to grain test with small clear specimens. a Front view, and $\mathbf{b}$ side view intercept of asymptote in a plastic region, $m_{1}$ represents a slope in a plastic region, and $\alpha$ and $\beta$ represent a coefficient used to increase the agreement around a yield point.

To apply the test results (relationship between stress $\sigma$ and strain $\varepsilon$ ) to the analyses of mechanical performance of the joint, it is required that geometrical condition of small clear specimens and loading condition are similar with the conditions applied to the joint. However, the similar conditions were difficult to recreate on compressive test. This research applied the results obtained by the compressive test shown in Fig. 4 to the mechanical analyses of the joint because the deformed mechanism in Fig. 4 is not so different with one in the joint and the test conditions is easy to set up.

\section{Results and discussion}

\section{Hysteresis characteristics of mortise-tenon joint}

Figure 5 shows an example of the relationship between moment resistance $M$ and deformation angle $\theta$ of column obtained by the mechanical test with mortise-tenon joints. This is the result from Specimen T-1 with a gap $g$ of $2.98 \mathrm{~mm}$ at the mortise-tenon joint. Figure 6 shows envelope curves taken from Fig. 5. The thin solid and dashed lines represent envelope curves for positive- and negative-direction loading, respectively, and the thick solid line represents the average moment resistance for these two envelope curves at the same deformation angle. The line shows that the sufficient moment resistance does not occur in the early stage of loading (at around $0.04 \mathrm{rad}$ or lower). This indicates that mortise and tenon do not sufficiently contact each other because of a gap at the joint, although a column is at a tilt. When the deformation angle subsequently exceeds $0.04 \mathrm{rad}$, the mortise and tenon gradually contact each other, and the moment resistance comes to occur. While the moment resistance grows as the

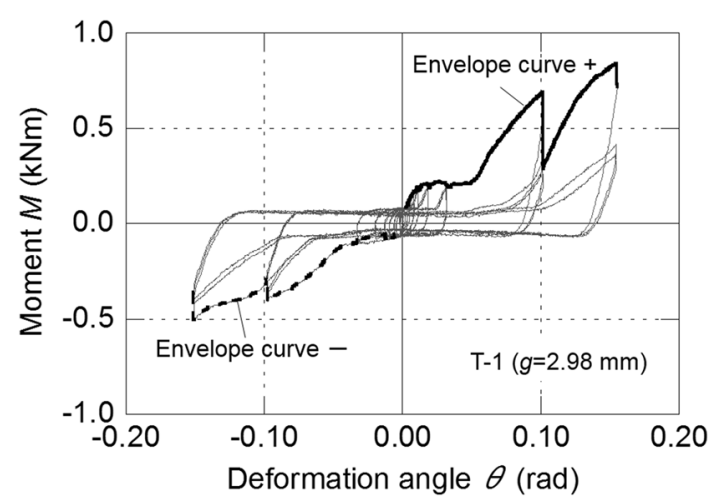

Fig. 5 Hysteresis characteristics of mortise-tenon joints 


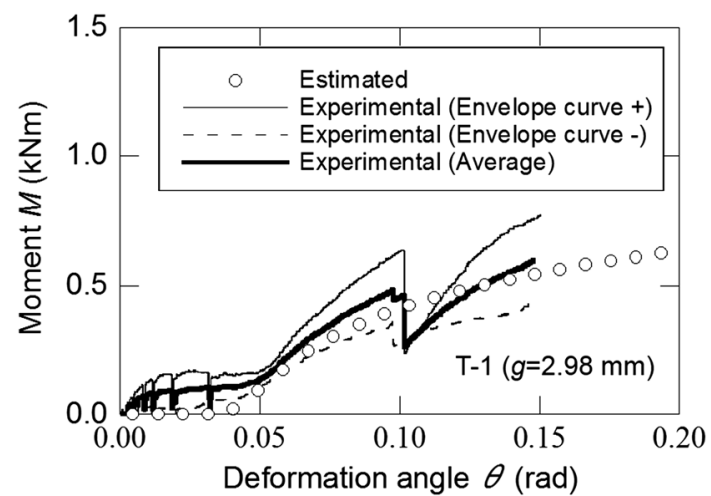

Fig. 6 Experimental and estimated results of mechanical test

deformation angle increases, it yields when the angle reaches around $0.07 \mathrm{rad}$, showing a rapid decrease before modest increase. After the test, the specimens were dismantled to observe its failure mode. As a result, while a compressive deformations were found at contact parts between a mortise and tenon and between beam and column perpendicular to grain, there were no breakages or other damages.

\section{Compressive behavior perpendicular to grain of small clear specimens}

Figure 7 shows one example of the relationship between stress $\sigma$ and strain $\varepsilon$ obtained by the compression perpendicular to grain test with the small clear specimens. The six thin lines in the figure represent results of six small clear specimens taken from a column of Specimen T-1, and the thick line represents the average stress for the six test results at the same strain. To express the thick line by Eq. (18), five parameters were determined. The square plots in the figure represent the relationship between stress and strain from Eq. (18) that were re-plotted with determined parameters. As these plots show the agreement with

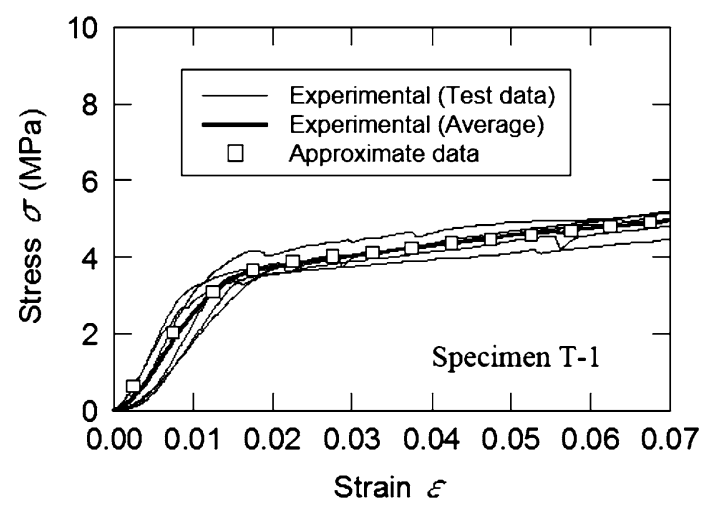

Fig. 7 Relationships between compressive stress and strain perpendicular to grain and approximation by Eq. (18)
Table 1 Parameters expressing the relationships between compressive stress and strain perpendicular to grain [Eq. (18)]

\begin{tabular}{lllllll}
\hline Specimen $^{\mathrm{a}}$ & $n^{\mathrm{b}}$ & $E_{\perp}(\mathrm{MPa})$ & $m_{0}(\mathrm{MPa})$ & $m_{1}(\mathrm{MPa})$ & $\alpha$ & $\beta$ \\
\hline $\mathrm{T}-1$ & 6 & 254.5 & 3.41 & 22.4 & 208.6 & 2.2 \\
$\mathrm{~T}-2$ & 6 & 305.2 & 3.55 & 22.8 & 444.0 & 2.3 \\
$\mathrm{~T}-3$ & 6 & 340.2 & 4.07 & 19.9 & 387.2 & 2.3 \\
$\mathrm{~T}-4$ & 6 & 449.2 & 5.34 & 40.9 & 357.2 & 2.3 \\
$\mathrm{~T}-5$ & 6 & 266.0 & 3.75 & 32.4 & 17.4 & 1.7 \\
T-6 & 6 & 303.9 & 3.07 & 22.4 & 555.4 & 2.4 \\
\hline
\end{tabular}

a Joint specimen from which small clear specimens were taken

b Number of small clear specimens

the thick line of test results, it was decided to use the equation and these parameters for the estimation of the relationship between $M$ and $\theta$ at the mortise-tenon joints. Table 1 lists parameters with regard to compressive behavior of six small clear specimens which were taken each column of joint specimen.

\section{Estimation of $M-\theta$ relationship at mortise-tenon joints}

Round plots in Fig. 6 show results of estimation of the relationship between $M$ and $\theta$ at the mortise-tenon joints. These are results from Specimen T-1, and are obtained by Eq. (16) through Eq. (4) after expressing the relationship between compressive stress and strain perpendicular to grain by Eq. (18) with the parameters in Table 1. The plots show that the moment value is zero when the deformation angle is about $0.03 \mathrm{rad}$ or lower. This is because mortise and tenon, in terms of the mechanical model proposed, do not contact each other due to a gap at the joint, that is, there is no resistance. When the deformation angle reaches around $0.04 \mathrm{rad}$, the moment resistance comes to occur, while the moment resistance starts growing in an exponential manner with an increase in the deformation. Round plots are between thin solid and dashed lines of the test results, showing behavior as if in line with the thick solid line. As the estimations for the other five specimens (T-2T-6) generally agree with the test results, this analysis model which takes into consideration a gap at the joint is considered to have good capability to estimate the relationship between $M$ and $\theta$.

Next, to verify various aspects of the estimation method for mechanical behavior derived in this study, a change in mechanical properties associated with a change in gap $g$ was numerically analyzed. Figure 8 describes the properties analyzed; rotational stiffness, initial slip, and strain energy. The rotational stiffness is represented by a slope at the linear part of the relationship between $M$ and $\theta$, and the initial slip is an intersection where the initial slope representing the rotational stiffness meets a horizontal axis (that 


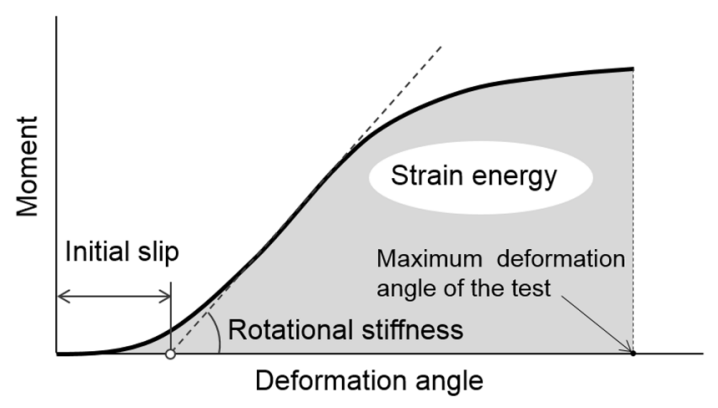

Fig. 8 Properties analyzed of mortise-tenon joints

is, the deformation angle that triggers the occurrence of sufficient moment resistance at the joints). For the strain energy, the area enclosed by $M-\theta$ curves up to the maximum deformation angle of the mechanical test $(1 / 6.5 \mathrm{rad})$ was calculated.

\section{Rotational stiffness}

Figure 9 shows the relationship between rotational stiffness and gap $g$. The vertical axis, which expresses the rotational stiffness, is normalized by the estimated rotational stiffness when the gap is $0.0 \mathrm{~mm}$. Six lines represent numerical analysis results for six joint specimens, while six plots constitute experimental results (average for each specimen under the positive- and negative-direction loading). Generally, it is expected that the greater a gap at the mortisetenon joints becomes, the lower the rotational stiffness is shown. Experimental results show that the rotational stiffness decreases by approximately $27 \%$ for $g$ of $1.0 \mathrm{~mm}$, and by $50 \%$ for $g$ of $3.0 \mathrm{~mm}$, making clear the large influence of a gap on a decrease in the rotational stiffness. On the other hand, the numerical analysis results also clearly indicate that the rotational stiffness declines with an increase in a size of gap $g$, with a decrease by

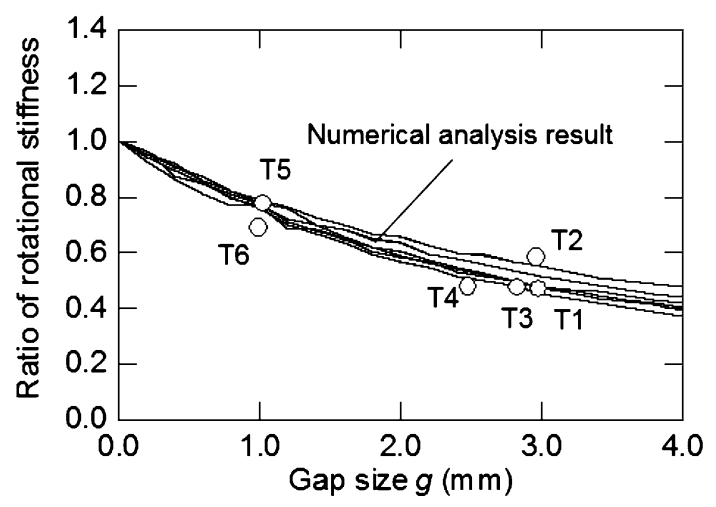

Fig. 9 Experimental and analytical results of rotational stiffness. The vertical axis is normalized by the estimated rotational stiffness when the gap is $0.0 \mathrm{~mm}$, while the horizontal axis expresses a gap $g$ between tenon and mortise approximately $22 \%$ for $g$ of $1.0 \mathrm{~mm}$ and $47 \%$ for $g$ of $3.0 \mathrm{~mm}$. Thus, as the analysis results generally agree with experimental results, use of a gap size as parameter enables to quantify a decrease in the rotational stiffness.

\section{Initial slip}

Figure 10 provides the relationship between initial slip and gap $g$. Plots of experimental results show the moment resistance occurs at the joints when the deformation angle reaches approximately $0.023 \mathrm{rad}$ for $g$ of $1.0 \mathrm{~mm}$ and $0.034 \mathrm{rad}$ for $g$ of $3.0 \mathrm{~mm}$. Numerical analysis results indicate that the six lines almost agree with each other, demonstrating that the initial slip goes up proportionately with an increase in a size of gap $g$, with the initial slip of approximately $0.014 \mathrm{rad}$ for $g$ of $1.0 \mathrm{~mm}$ and $0.039 \mathrm{rad}$ for $g$ of $3.0 \mathrm{~mm}$. Compared with the experimental results, the numerical analysis results generally show the agreement with them, with a value being underpredicted in the range of small $g$ and overpredicted in the range of large $g$. The numerical analysis results show that an influence of difference in compressive properties perpendicular to grain (Table 1) on the initial slip is smaller than that on the rotational stiffness. It is considered that this is because the initial slip is dominantly influenced by the geometric arrangement of mortise and tenon (whether tenon and mortise contact each other or not) when the joints deform (or columns are at a tilt).

\section{Strain energy}

Figure 11 describes the relationship between strain energy and a gap $g$. The vertical axis expresses the strain energy normalized by its estimation when $g$ is $0.0 \mathrm{~mm}$. Plots in the figure constitute experimental results (average for each specimen under the positive- and negative-direction loading). The figure shows that the strain energy decreases by approximately $13 \%$ for $g$ of $1.0 \mathrm{~mm}$ and by $47 \%$ for $g$ of

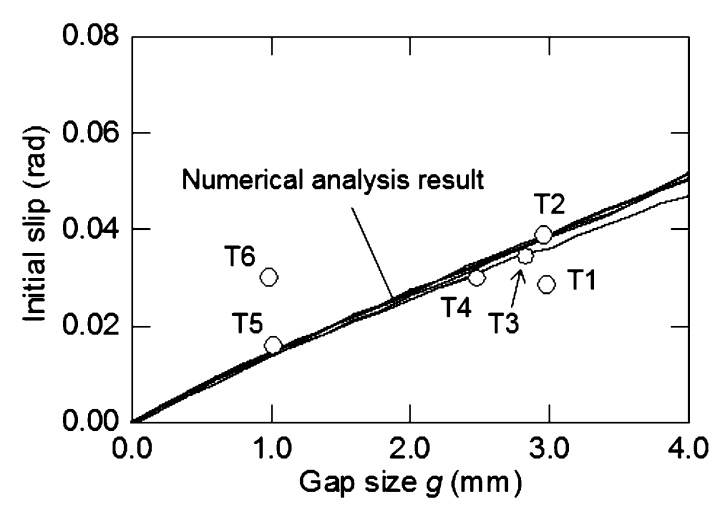

Fig. 10 Experimental and analytical results of initial slip 


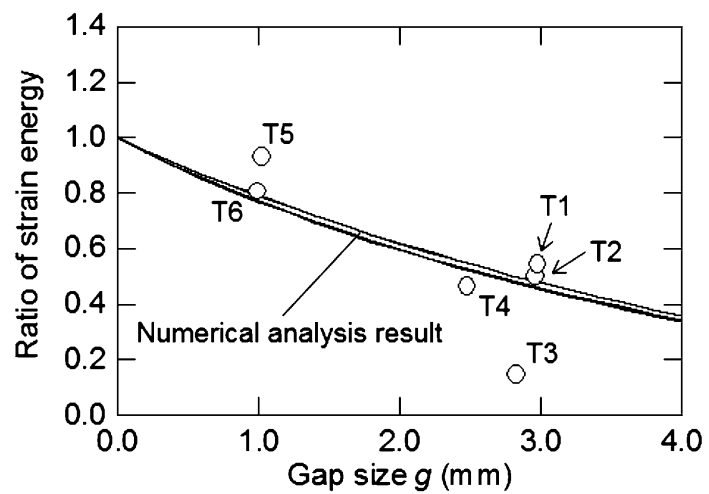

Fig. 11 Experimental and analytical results of strain energy. The vertical axis is normalized by the estimated strain energy when the gap is $0.0 \mathrm{~mm}$, while the horizontal axis expresses a gap $g$ between tenon and mortise

$3.0 \mathrm{~mm}$. (However, as it decreases by approximately $85 \%$ for the specimen T3, an outlier may appear.) The numerical analysis results are expressed almost by one line, with the strain energy decreasing by $21 \%$ for $g$ of $1.0 \mathrm{~mm}$ and by $52 \%$ for $g$ of $3.0 \mathrm{~mm}$. Compared with experimental results, the numerical analysis results generally show the agreement with them, although consideration should be given to the possible appearance of a plot well below the analysis results (Specimen T3). Like the initial slip, the analysis of the strain energy finds the little influence of a difference in partial compressive properties perpendicular to grain (Table 1).

\section{Conclusion}

This study theoretically and experimentally analyzed the mechanical performance of mortise-tenon joints involving a gap. First, a method to estimate the moment resistance and deformation angle of column at the joints was theoretically derived. According to the estimation, the moment resistance does not occur in the range of small deformation angle if the mortise-tenon joints involve a gap. After that, the moment resistance comes to occur with growing proportionately with an increase in deformation. After yield, it modestly increases. Compared with experimental results, the estimated results generally show the agreement with them, indicating the validity of the estimation method derived. Furthermore, the derived estimation method was used to numerically analyze to determine the relationship between gap size and mechanical properties (rotational stiffness, initial slip and strain energy). As a result, a gradual decrease in the mechanical performance of the joints with the increase in size of the gap was quantified, indicating the importance of giving consideration to the gap in evaluating the mechanical performance of the joints.

\section{Appendix}

Lines $2-8$ in Fig. 2, based on their geometric relationship, are obtained as follows:

Line 2: $y=-\tan \theta \cdot x-\frac{l}{2 \cos \theta}+Y$

Line 3: $y=\frac{1}{\tan \theta} x-\frac{h-g}{2 \sin \theta}+Y$

Line 4: $y=\frac{1}{\tan \theta} x+\frac{h-g}{2 \sin \theta}+Y \quad$ Line 5: $\quad y=\frac{l}{2}$

Line 6 : $x=-\frac{h}{2} \quad$ Line $7: \quad x=\frac{h}{2}$

Line 8: $y=\frac{1}{\tan \theta} x-\frac{h_{c}}{2 \sin \theta}+Y$

In addition, by solving a simultaneous equation with function equations for Lines 1-8, the coordinates of Intersections $\mathrm{A}-\mathrm{H}$ are calculated as follows:

$$
\begin{aligned}
\mathrm{A}\left(A_{x}, A_{y}\right)= & \left\{-\frac{h}{2},-\frac{h-g}{2}\left(\frac{1}{\tan \theta}-\frac{1}{\sin \theta}\right)-\frac{g}{2 \tan \theta}+Y\right\} \\
\mathrm{B}\left(B_{x}, B_{y}\right)= & {\left[-\frac{1}{2}\{l \sin \theta+(h-g) \cos \theta\},\right.} \\
& \left.\frac{1}{2}\{l \sin \theta \tan \theta+(h-g) \sin \theta\}-\frac{l}{2 \cos \theta}+Y\right] \\
\mathrm{C}\left(C_{x}, C_{y}\right)= & \left(-\frac{h}{2}, \frac{h}{2} \tan \theta-\frac{l}{2 \cos \theta}+Y\right) \\
\mathrm{D}\left(D_{x}, D_{y}\right)= & \left\{\frac{h}{2}, \frac{h-g}{2}\left(\frac{1}{\tan \theta}-\frac{1}{\sin \theta}\right)+\frac{g}{2 \tan \theta}+Y\right\} \\
\mathrm{E}\left(E_{x}, E_{y}\right)= & {\left[\frac{1}{2}\{l \sin \theta+(h-g) \cos \theta\},\right.} \\
& \left.-\frac{1}{2}\{l \sin \theta \tan \theta+(h-g) \sin \theta\}+\frac{l}{2 \cos \theta}+Y\right] \\
\mathrm{F}\left(F_{x}, F_{y}\right)= & \left(\frac{h}{2},-\frac{h}{2} \tan \theta+\frac{l}{2 \cos \theta}+Y\right) \\
\mathrm{G}\left(G_{x}, G_{y}\right)= & {\left[\frac{1}{\tan \theta}\left\{\frac{l}{2}\left(\frac{1}{\cos \theta}-1\right)+Y\right\}, \frac{l}{2}\right] } \\
\mathrm{H}\left(H_{x}, H_{y}\right)= & {\left[\frac{1}{2}\left\{l \sin \theta+h_{c} \cos \theta\right\},\right.} \\
& \left.-\frac{1}{2}\left\{l \sin \theta \tan \theta+h_{c} \sin \theta\right\}+\frac{l}{2 \cos \theta}+Y\right]
\end{aligned}
$$

\section{References}

1. Suzuki Y, Maeno M (2006) Structural mechanism of traditional wooden frame by dynamic and static tests. Struct Control Health Monit 13:508-522

2. Wang H, Scanlon A, Shang S, He F (2013) Comparison of seismic experiments on traditional Chinese wood structures and light wood-framed structure. J Struct Eng 139:2038-2043

3. Guan ZW, Kitamori A, Komatsu K (2008) Experimental study and finite element modelling of Japanese "Nuki" joints part two: 
racking resistance subjected to different wedge configurations. Eng Struct 30:2041-2049

4. Chang WS, Hsu MF, Komatsu K (2006) Rotational performance of traditional Nuki joints with gap I: theory and verification. J Wood Sci 52:58-62

5. Eckelman CA, Haviarova E (2011) Rectangular mortise and fullwidth tenon joints in ready-to-assemble light-frame timber constructions. Wood Fiber Sci 43:346-352

6. Nishimura T, Goto M, Suzuki Y (2010) An experimental study on the stress transfer in mortise-tenon joints for wooden frame structures (in Japanese). J Struct Constr Eng AIJ 75:2197-2204

7. Sakata H, Yamazaki Y, Udagawa H, Ohashi Y (2012) Experimental study on flexural-Shear behavior of mortise-tenon joint with dowel (in Japanese). J Struct Constr Eng AIJ 77:45-54
8. Tanahashi H, Suzuki Y (2012) Basic concept and general formulation of restoring Force characteristics of traditional wooden joints. In: Proceedings of the 13th World Conference on Timber Engineering, July 16-19, 2012, Auckland, New Zealand, pp 378-387

9. Japan housing And Wood Technology Center (2008) Allowable stress design for wooden Framework method (in Japanese). Japan Housing And Wood Technology Center, Tokyo

10. Tanahashi H, Ooka Y, Izuno K, Suzuki Y (2011) Yielding mechanism of embedment of wood and formulation of elastoplastic embedded displacements (in Japanese). J Struct Constr Eng AIJ 76:811-819

11. Sasaki Y, Miura S, Takemura T (1988) Non-linear analysis of a semi-rigid jointed metal plate wood-truss. Mokuzai Gakkaishi 34: $120-125$ 